\title{
Clinical characteristics of critically ill patients with 2019 novel coronavirus (COVID-19): do we need a new triage system?
}

\author{
Mehmet Akif YAZAR ${ }^{1, *}{ }_{\odot}$, Yasin TIRE ${ }^{1}$, Fatih YUCEL ${ }^{2}$, Hasan SENAY ${ }^{2}$, Ercan KURTIPEK ${ }^{3}$, \\ Nevin SEKMENLI ${ }^{4}$, Guzide YAZAR ${ }^{4}$
}

${ }^{1}$ Department of Anesthesiology and Reanimation, University of Health Sciences, Konya Training and Research Hospital, 42040 Konya, Turkey ${ }^{2}$ Department of Intensive Care, University of Health Sciences, Konya Training and Research Hospital,42040 Konya, Turkey

${ }^{3}$ Department of Chest Diseases, University of Health Sciences, Konya Training and Research Hospital,42040 Konya, Turkey

${ }^{4}$ Department of Radiology, University of Health Sciences, Konya Training and Research Hospital,42040 Konya, Turkey

*Correspondence

makifyazar@hotmail.com

(Mehmet Akif YAZAR)

\begin{abstract}
Background: This expanded study presents the characteristic features of patients with novel Coronavirus 2019 (COVID-19) in intensive care units (ICUs). On the other hand, it has revealed an issue of triage on admission to ICUs for patients with COVID-19.

Methods: The critically ill patients' characteristics, laboratory findings, treatment and outcomes data were recorded. All chest computed tomography (c-CT) images were reviewed by two experienced radiologists in chest imaging. Collected data were compared between the confirmed and suspected COVID-19 cases. Moreover, some detected parameters were evaluated via c-CT findings among suspected COVID-19 cases.

Results: The study population included 105 patients hospitalized in ICUs. Twenty-seven patients $(25.7 \%)$ were confirmed COVID-19 through real-time reverse-transcription polymerase-chain-reaction (RT-PCR) assay, and 78 patients (74.3\%) were suspected COVID-19. There was a significant difference between the confirmed COVID-19 and suspected COVID-19 patients in terms of $\mathrm{PaO}_{2} / \mathrm{FiO}_{2}$ ratio, APACHE II scoring system, the number of comorbidities. Interestingly, in suspected cases, mean $\mathrm{PaO}_{2} / \mathrm{FiO}_{2}$ ratio, APACHE II score, and the number of comorbidities were significantly higher in patients with typical c-CT findings for COVID-19 $(P=0.038, P=0.034$ and $P=0.020$, respectively). Considering all three parameters, $33.3 \%$ of cases with typical CT findings could be reconsidered as highly probable COVID-19 infections. Moreover, $16.7 \%$ of the cases with atypical CT findings could be excluded and the unnecessary burden on ICUs could be reduced.

Conclusion: In many contagious diseases such as COVID-19, for a new triage system, specific characteristics, selected general physiological findings, and typical laboratory parameters may be standardized in addition to RT-PCR testing and c-CT examination.
\end{abstract}

\section{Keywords}

Coronavirus; COVID-19; SARS CoV-2; Intensive care unit; Critical illness; Triage

\section{Introduction}

In December 2019, cases of pneumonia of unknown etiology began emerging in Wuhan, China. On February 11, 2020, the disease-identified as severe acute respiratory syndrome coronavirus-2 (SARS-CoV-2) - was named COVID-19 by the World Health Organization (WHO). Since then, the disease has continued to spread rapidly from person to person and become a global pandemic. As of May 18, 2020, 4,618,821 cases were detected and 311,847 deaths had been reported [1].

We now know that the infection is transmitted person-toperson through droplets, contact, and aerosol transmission generated during coughing and sneezing by symptomatic patients, but that it can also occur from contact with asymptomatic people and before the onset of symptoms [2, 3]. Many stud- ies have stated that patients' clinical manifestations included fever, non-productive cough, dyspnea, myalgia, and fatigue [4-6]. It seems that age, biological sex, and comorbid diseases such as hypertension, diabetes, and chronic lung diseases worsen the progress of COVID-19 [7-9]. Individuals with multiple comorbidities are prone to severe infection and may also present with acute respiratory distress syndrome (ARDS) and acute kidney injury (AKI) [10]. Recently, the majority of COVID-19 patients with comorbid diseases have been treated in intensive care units (ICUs). Unfortunately, some of them have died due to complications [11].

According to the WHO guidelines for diagnosis and treatment of 2019 novel coronavirus (2019-nCoV), routine confirmation of cases of COVID-19 is based on the detection of unique sequences of virus RNA by nucleic acid amplification 
tests such as real-time reverse-transcription polymerase-chainreaction (RT-PCR) [12]. On the other hand, chest computed tomography (c-CT) imaging has become a valuable screening tool with high sensitivity and specificity in the diagnosis of patients with COVID-19 [13].

The global pandemic has caused an unprecedented number of critically ill COVID-19 patients. Intensivists struggle to manage these patients when resources are insufficient or even completely lacking. In fact, ICU admissions are dependent on both the severity of illness and the ICU capacity of the healthcare system. Therefore, many patients with either confirmed or suspected COVID-19 have been hospitalized in ICUs with a limited number of beds. This ambiguity may result in both a contamination risk to uninfected patients and the unnecessary occupancy of limited ICU beds. As a result, it may be necessary to create a new triage system for these contagious critically ill patients.

In this study, the epidemiological and clinical characteristics of suspected or confirmed COVID-19 patients admitted to the intensive care unit were described and compared with each other. According to the results, it was aimed to develop a new triage system that gives priority to patients with the right criteria for admission to the intensive care unit in cases of epidemic diseases.

\section{Materials and methods}

\subsection{Study design and participants}

This retrospective study was approved by the local ethics committee of Selcuk University, Konya (Acceptance number: 2020/245). The study included both confirmed and suspected COVID-19 patients hospitalized in ICUs allocated for patients with novel coronavirus disease in Konya Training and Research Hospital from March 15-May 15, 2020. Patients who had positive RT-PCR testing were accepted as confirmed COVID-19 cases. The suspected COVID-19 cases were defined as those whose RT-PCR analyses were negative, but who had the typical symptoms of the novel coronavirus disease, such as cough, high fever $\left(>38.5^{\circ} \mathrm{C}\right)$, or dyspnea, and those with a history of contact with a confirmed COVID-19 patient in addition to typical symptoms. Suspected COVID-19 cases had also at least one of the following laboratory findings in addition to typical symptoms: typical or atypical c-CT finding, high level of serum ferritin $(>200 \mathrm{ng} / \mathrm{mL})$, D-dimer $(>500$ $\mu \mathrm{g} / \mathrm{L})$, C-reactive protein $(>10 \mathrm{mg} / \mathrm{L})$ and lymphopenia $(<$ $1.10^{3} / \mu \mathrm{L}$ ). The clinical outcomes (i.e., discharges, mortality, and length of stay) were monitored up to May 15, 2020, the final date of follow-up. Collected data were compared between the confirmed and suspected COVID-19 cases. Moreover, some detected parameters were evaluated through c-CT findings in suspected COVID-19 cases. Patients with longer than seven days' time-interval between c-CT imaging and the RTPCR assay and with cardiac arrest immediately after admission to the ICU were excluded from the study.

\subsection{Data collection}

The patients' epidemiological, clinical, laboratory and radiological characteristics and treatment and outcomes data were accessed from the Hospital Data Management System (HDMS) of Konya Training and Research Hospital and Public Health Management System (PHMS) of the Ministry of Health, Turkey. The information recorded included demographic data, infected medical staff, medical history, exposure history, preexisting comorbidities, signs and symptoms (fever $>37.5{ }^{\circ} \mathrm{C}$, cough, shortness of breath, weakness, and muscle soreness), laboratory parameters reflecting COVID-19 pneumonia (i.e., hemogram, white blood cell [WBC] count, C-reactive protein [CRP], procalcitonin, lymphocyte, D-dimer and ferritin), c-CT scan findings, and treatment measures (medical drug therapy, respiratory support, kidney replacement therapy). Acute Physiology and Chronic Health Evaluation II (APACHE II) scores and Glasgow Coma Scale (GCS) were determined on the day of ICU admission. Ratio of the partial pressure of arterial oxygen to fraction of inspired oxygen $\left(\mathrm{PaO}_{2} / \mathrm{FiO}_{2}\right)$ was noted when patients were admitted to ICU. The first vital signs and laboratory findings on admission were not shown separately since they were enrolled as APACHE II scoring system. Comorbidities were initially noted as a categorical variable (yes vs. no) and quantity. Furthermore, comorbidities were classified according to the organ systems (i.e., respiratory, cardiovascular, or endocrine). The comorbidity conditions were determined based on HDMS and patient's self-report on admission. Complications such as ARDS and AKI developed during treatment were noted. ARDS was defined according to the Berlin definition [14]. AKI was identified according to the Kidney Disease: Improving Global Outcomes definition [15].

\subsection{Real-time reverse transcription polymerase chain reaction assay for COVID-19}

Real-time reverse-transcription polymerase chain reaction (RT-PCR) assay of a combined nasal-throat swab was performed on patients within the first 12 hours of admission to the ICU. The RT-PCR results were extracted from patients' electronic medical records in PHMS. Patients with positive RT-PCR tests were accepted as confirmed COVID-19 cases. In accordance with the COVID-19 Guidelines of General Directorate of Public Health, Turkey, the control samples were taken five days later to evaluate the response to treatment of patients with a positive RT-PCR test, and 24 hours later to rule out a negative RT-PCR test despite significant clinical findings.

\subsection{Interpretation of chest computed tomography imaging}

Chest computed tomography (c-CT) imaging analysis was performed using the hospital digital database system. All c-CT images were reviewed by two radiologists (G.Y. and N.S.), with eight and 15 years of experience in chest imaging, respectively. Imaging was reviewed independently and final decisions reached by consensus.

The c-CT scan was evaluated for subpleural/peripheral or peribronchovascular distribution, ground-glass opacities or consolidation, air bronchogram, interlobular septal thickening, cavitation, fibrotic lesions, centrilobular nodules, pleural 
effusion, thoracic lymphadenopathy, and underlying lung diseases such as tuberculosis, emphysema, or interstitial lung. Ground-glass opacification was defined as hazy increased lung attenuation with preservation of bronchial and vascular margins, and consolidation was defined as high-density patchy opacities, inside which air bronchograms [16]. Finally, radiologists classified their interpretations into two groups, as follows:

(1) Typical c-CT findings (typical findings consistent with COVID-19 pneumonia): The typical c-CT findings defined as bilateral, subpleural and peripheral ground-glass opacities, crazy-paving appearance, air space consolidation, bronchovascular thickening, and traction bronchiectasis [11, 17].

(2) Atypical c-CT findings (atypical pneumonia findings in accordance with either viral or bacterial pneumonia): The atypical c-CT findings were defined as thickening and plugs on the branch wall, centrilobular nodules and tree-in-bud appearance, mosaic perfusion and/or air trapping, symmetrical or diffuse ground-glass opacities, and consolidation [18].

\subsection{Statistical analysis}

Categorical variables were described as frequency rates and percentages, and continuous variables were described using mean (SD) or median (IQR) values. Means for continuous variables were compared using an independent $t$-test when the data were normally distributed; otherwise, the Mann-Whitney test was used. Proportions for categorical variables were compared using the $x^{2}$ test. Statistical analysis was performed using IBM SPSS Statistics for Windows, Version 22.0 (IBM Corp.). For unadjusted comparisons, a two-sided $\alpha$ of less than 0.05 was considered statistically significant.

\section{Results}

\subsection{Demographic and clinical characteristics}

The study population included 105 patients hospitalized in ICUs. Twenty-seven patients $(25.7 \%)$ were confirmed COVID-19 through RT-PCR assay, and 78 patients (74.3\%) were suspected COVID-19. Of the confirmed COVID-19 cases, four patients $(14.8 \%)$ had a negative result with RT-PCR on admission. Eleven patients' test results (40.7\%) converted from positive to negative during the hospital stay.

The median age of all patients $(\mathrm{N}=105)$ was 70 years (IQR, 58.5-80.0). No infected medical staff $(0 \%)$ was among the critically ill patients. The most common symptom was shortness of breath, and the least common symptom was cough. Of the 105 patients, 96 (91.4\%) had one or more comorbid diseases. The most common pre-existing comorbid diseases were diabetes $(36.2 \%)$, hypertension $(29.5 \%)$, and chronic obstructive pulmonary disease (18.1\%). Demographic features and baseline data for all patients are shown in Table 1.

There was a significant difference between the confirmed COVID-19 and suspected COVID-19 patients in terms of $\mathrm{PaO}_{2} / \mathrm{FiO}_{2}$ ratio, APACHE II scoring system, the number of comorbidities, and the source of transmission.

\subsection{Laboratory parameters and radiologic findings}

There was no significant difference between the confirmed COVID-19 and suspected COVID-19 patients in terms of hemoglobin, hematocrit values, and some infection parameters. Compared with suspected COVID-19 patients, the patients with confirmed COVID-19 had lower lymphocyte counts $(0.98 \pm 0.51, P=0.005)$. WBC count was normal range (4.5$12.6 \times 103)$ in the confirmed COVID-19 cases and above the normal range in the suspected COVID-19 cases, but there was no significant difference between the confirmed and suspected COVID-19 cases. Laboratory parameters of COVID-19 pneumonia are shown in Table 2.

Of the 105 patients, 63 (60\%) had typical c-CT findings for COVID-19. c-CT findings for all confirmed COVID-19 cases $(27,100 \%)$ were compatible with typical c-CT findings (Table 3). Four cases initially missed by the RT-PCR testing had been detected by the c-CT examination at the first admission. Of 78 suspected COVID-19 cases, 36 (46.2\%) had typical cCT findings for the disease. c-CT images of six critically ill patients are shown in Fig. 1.

Ninety-nine critically ill patients $(94.3 \%)$ received antiviral treatment in accordance with the COVID-19 Guidelines of General Directorate of Public Health, Turkey. Seventeen patients $(16.2 \%)$ were administered corticosteroid therapy (methylprednisolone 40-120 mg per day) as 1-2 mg/kg/day, 57 days in ARDS cases with mechanical ventilation. Thirty-four patients (32.4\%) received antibiotic therapy.

Fifty-four patients (51.4\%) were intubated and invasive mechanical ventilation (IMV) was required. Sixty-four patients $(61.5 \%)$ required non-invasive mechanical ventilation (NIMV) and $15(14.3 \%)$ patients received higher-level oxygen support through high-flow nasal cannula (HFNC). Six patients (5.7\%) received only oxygen with a simple nasal cannula. Ten patients $(9.5 \%)$ required renal replacement therapy. The most common complication was ARDS (19\%). Compared with suspected COVID-19 cases, ARDS was significantly more common in confirmed COVID-19 cases.

By the end of May 15, 2020, 25 patients (23.8\%) had been discharged and 64 patients $(61 \%)$ had died. A significantly higher number of deaths occurred among confirmed COVID19 cases. The median age of the patients who died with confirmed and suspected COVID-19 was 68 years (IQR, 58.5075.50) and 72 years (IQR, 61.00-81.00), respectively. All treatments, complications and clinical outcomes of critically ill patients are shown in Table 4.

Mean $\mathrm{PaO}_{2} / \mathrm{FiO}_{2}$ ratio, APACHE II score, and the number of comorbidities were significantly higher in patients with typical c-CT findings $(P=0.038, P=0.034$ and $P=0.020$, respectively). Considering all three parameters, 12 patients $(33.3 \%)$ with typical c-CT findings were beyond the mean values of parameters. Likewise, seven patients (16.7\%) with atypical c-CT findings were beyond the mean values of parameters. A comparison of these parameters with c-CT findings for suspected COVID-19 patients is shown in Table 5. 
TA B L E 1. Demographic features and baseline data of critically ill patients.

\begin{tabular}{|c|c|c|c|c|}
\hline Characteristics & All patients $(\mathrm{N}=105)$ & $\begin{array}{l}\text { Confirmed COVID-19 } \\
(\mathrm{N}=27)\end{array}$ & $\begin{array}{l}\text { Suspected COVID-19 } \\
(\mathrm{N}=78)\end{array}$ & $P$-value \\
\hline Age, median (IQR), y & $70(58.5-80)$ & $68(59-75)$ & $72.5(57-80)$ & 0.913 \\
\hline \multicolumn{5}{|l|}{ Sex } \\
\hline Male & $57(54.2 \%)$ & $13(48.1 \%)$ & $44(56.4 \%)$ & 0.458 \\
\hline Female & $48(45.7 \%)$ & $14(51.8 \%)$ & $34(43.6 \%)$ & \\
\hline Infected medical staff & $0(0 \%)$ & $0(0 \%)$ & $0(0 \%)$ & - \\
\hline \multicolumn{5}{|l|}{ Signs and symptoms } \\
\hline Fever $\left(>37.5^{\circ} \mathrm{C}\right)$ & $28(26.7 \%)$ & $7(25.9 \%)$ & $21(26.9 \%)$ & 0.920 \\
\hline Cough & $21(20 \%)$ & $6(22.2 \%)$ & $15(19.2 \%)$ & 0.738 \\
\hline Shortness of breath & $85(81 \%)$ & $21(77.8 \%)$ & $64(82.1 \%)$ & 0.626 \\
\hline Weakness & $36(34.3 \%)$ & $10(37 \%)$ & $26(33.3 \%)$ & 0.727 \\
\hline Muscle soreness & $26(24.8 \%)$ & $8(29.6 \%)$ & $18(23.1 \%)$ & 0.497 \\
\hline $\mathrm{PaO}_{2} / \mathrm{FiO}_{2}$ ratio, mmHg, mean $( \pm \mathrm{SD})$ & $182.5( \pm 80.8)$ & $156.3( \pm 71.6)$ & $191.5( \pm 82.2)$ & $0.039 *$ \\
\hline APACHE-II, mean $( \pm \mathrm{SD})$ & $25.55( \pm 7.92)$ & $28.22( \pm 9.26)$ & $24.62( \pm 7.24)$ & $0.042 *$ \\
\hline GCS, mean (SD) & $8.38(4.24 \%)$ & $7.96(3.94 \%)$ & $8.53(4.35 \%)$ & 0.555 \\
\hline Current smoking & $28(26.7 \%)$ & $6(22.2 \%)$ & $22(28.2 \%)$ & 0.545 \\
\hline Comorbidity presence & $96(91.4 \%)$ & $26(96.3 \%)$ & $70(89.7 \%)$ & 0.441 \\
\hline The number of Comorbidity, mean $( \pm \mathrm{SD})$ & $1.51( \pm 1.15)$ & $2( \pm 1.03)$ & $1.48( \pm 1.11)$ & $0.038^{*}$ \\
\hline Cardiovascular diseases & $38(36.2 \%)$ & $11(39.3 \%)$ & $27(35.1 \%)$ & 0.691 \\
\hline Hypertension & $31(29.5 \%)$ & $10(35.7 \%)$ & $21(27.3 \%)$ & 0.402 \\
\hline Coronary heart disease & $16(15.2 \%)$ & $3(10.7 \%)$ & $13(16.9 \%)$ & 0.550 \\
\hline Congestive heart failure & $8(7.6 \%)$ & $3(10.7 \%)$ & $5(6.4 \%)$ & 0.471 \\
\hline Respiratory diseases ${ }^{\dagger}$ & $23(21.9 \%)$ & $3(10.7 \%)$ & $20(25.6 \%)$ & 0.120 \\
\hline COPD & $19(18.1 \%)$ & $3(10.7 \%)$ & $16(20.8 \%)$ & 0.236 \\
\hline Malignancies & $8(7.6 \%)$ & $1(3.6 \%)$ & $7(9.1 \%)$ & $>0.99$ \\
\hline Lung & $4(3.8 \%)$ & $0(0 \%)$ & $4(5.2 \%)$ & 0.572 \\
\hline Liver & $2(1.9 \%)$ & $0(0 \%)$ & $2(2.6 \%)$ & $>0.99$ \\
\hline Multiple myeloma & $1(1 \%)$ & $1(3.6 \%)$ & $0(0 \%)$ & 0.267 \\
\hline Lymphoma & $1(1 \%)$ & $0(0 \%)$ & $1(1.3 \%)$ & $>0.99$ \\
\hline Endocrine disease (diabetes) & $38(36.2 \%)$ & $11(39.3 \%)$ & $27(35.1 \%)$ & 0.691 \\
\hline Chronic renal disease & $9(8.6 \%)$ & $1(3.6 \%)$ & $8(10.4 \%)$ & 0.439 \\
\hline Immunodeficiency & $2(1.9 \%)$ & $0(0 \%)$ & $2(2.6 \%)$ & $>0.99$ \\
\hline Cerebrovascular disease & $3(2.9 \%)$ & $0(0 \%)$ & $3(3.9 \%)$ & 0.563 \\
\hline \multicolumn{5}{|l|}{ Source of transmission } \\
\hline Domestic & $81(77.1 \%)$ & $16(59.3 \%)$ & $65(83.3 \%)$ & $0.016^{*}$ \\
\hline Foreign & $24(22.9 \%)$ & $13(48.1 \%)$ & $11(14.1 \%)$ & \\
\hline Length of stay, mean, (SD), d & $7.58( \pm 5.68)$ & $9.03( \pm 7.63)$ & $7.07( \pm 4.78)$ & 0.220 \\
\hline
\end{tabular}

Data are presented as median (IQR $=$ interquartile range) or mean $(S D=$ standard deviation) values. Proportions were compared using the $x^{2}$ test or Fisher exact test for categorical variables and independent group t-tests for continuous variables. $P<0.05$ was considered statistically significant. Abbreviations: COPD, chronic obstructive pulmonary disease; GCS, Glasgow coma scale.

* $P$ values $<0.05$.

${ }^{\dagger}$ Respiratory diseases were included COPD and lung malignancy.

\section{Discussion}

While this expanded retrospective study, on the one hand, presents the characteristic features of patients with COVID-
19 in the ICU, on the other hand, it has revealed an issue of triage on admission to ICUs for patients with contagious 
TA B L E 2. Laboratory parameters of critically ill patients.

\begin{tabular}{|llllc} 
& All patients $(\mathrm{N}=105)$ & $\begin{array}{l}\text { Confirmed COVID-19 } \\
(\mathrm{N}=27)\end{array}$ & $\begin{array}{l}\text { Suspected COVID-19 } \\
(\mathrm{N}=78)\end{array}$ & $P$-value \\
\hline $\begin{array}{l}\text { Laboratory parameters, mean }( \pm \mathrm{SD}) \\
\text { Hemoglobin }(\mathrm{g} / \mathrm{dL})\end{array}$ & $11.28( \pm 2.04)$ & $11.62( \pm 2.47)$ & $11.16( \pm 1.87)$ & 0.309 \\
\hline Hematocrit $(\%)$ & $33.32( \pm 5.23)$ & $34.46( \pm 6.07)$ & $32.92( \pm 4.89)$ & 0.189 \\
\hline Lymphocyte $\left(10^{3} / \mu \mathrm{L}\right)$ & $1.32( \pm 1.30)$ & $0.98( \pm 0.51)$ & $1.44( \pm 1.47)$ & $0.021^{*}$ \\
\hline $\mathrm{WBC}\left(10^{3} / \mu \mathrm{L}\right)$ & $12.74( \pm 6.13)$ & $11.64( \pm 4.76)$ & $13.12( \pm 6.52)$ & 0.284 \\
\hline $\mathrm{CRP}(\mathrm{mg} / \mathrm{L})$ & $104.28( \pm 81.37)$ & $100.82( \pm 56.50)$ & $105.48( \pm 88.65)$ & 0.799 \\
\hline Procalcitonin $(\mathrm{ng} / \mathrm{mL})$ & $4.43( \pm 15.31)$ & $5.86( \pm 22.11)$ & $3.93( \pm 12.27)$ & 0.573 \\
\hline D-dimer $(\mu \mathrm{g} / \mathrm{L})$ & $350( \pm 501)$ & $236( \pm 324)$ & $390( \pm 546)$ & 0.170 \\
\hline Ferritin $(\mathrm{ng} / \mathrm{mL})$ & $528.49( \pm 748.23)$ & $681.79( \pm 375.09)$ & $475.42( \pm 835.20)$ & 0.218 \\
\hline
\end{tabular}

Data are presented as median (IQR $=$ interquartile range) or mean $(S D=$ standard deviation $)$ values. Proportions were compared using the $x^{2}$ test or Fisher exact test for categorical variables and independent group t-tests for continuous variables. $P<0.05$ was considered statistically significant. Abbreviation: $C R P, C$-reactive protein; WBC, white blood cell. ${ }^{*} P$ values $<$ 0.05 .

TA B L E 3. Ratios of typical and atypical chest computed tomography findings for critically ill patients.

\begin{tabular}{|c|c|c|c|}
\hline & All patients $(\mathrm{N}=105)$ & Confirmed COVID-19 $(\mathrm{N}=27)$ & Suspected COVID-19 $(\mathrm{N}=78)$ \\
\hline \multicolumn{4}{|c|}{ c-CT Findings } \\
\hline Typical & $63(60 \%)$ & $27(100 \%)$ & $36(46.2 \%)$ \\
\hline Atypical & $42(40 \%)$ & $0(0 \%)$ & $42(53.8 \%)$ \\
\hline
\end{tabular}

Abbreviation: c-CT, chest computed tomography.
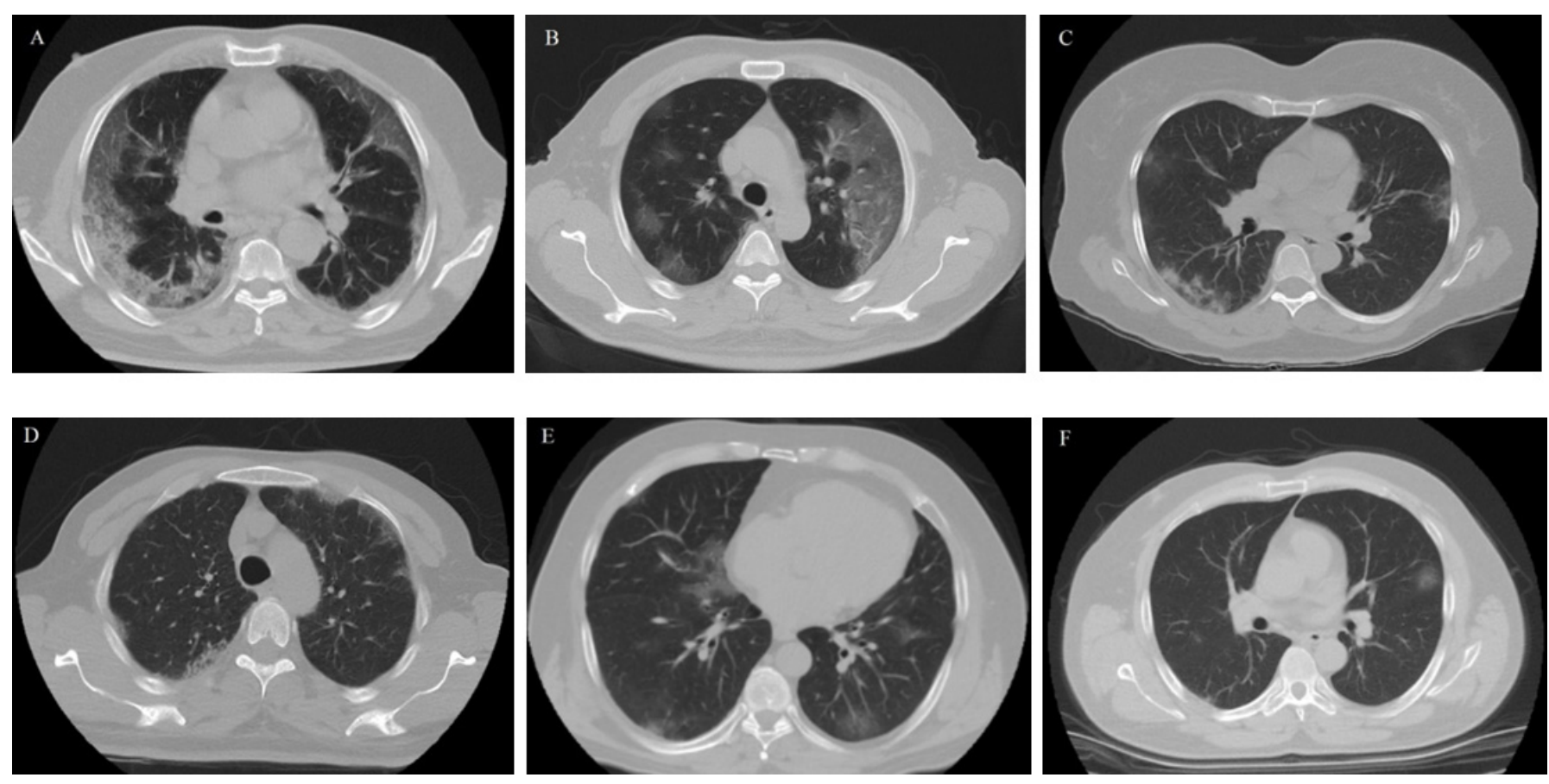

F I G U R E 1. Chest CT images of six critically ill patients. (Case A,B) Chest CT image sections from a 70 and 59-year-old men with confirmed COVID-19, respectively. Their c-CT images showed bilateral multilobular ground-glass density areas, subpleural interlobular septal thickening and consolidation areas in the lower lobes. (Case C,D) Chest CT image sections from a 50-year-old woman and 63-year-old man with negative RT-PCR test over the hospital stay, respectively. $\mathrm{PaO}_{2} / \mathrm{FiO}_{2}$ ratios of both were very low (131 and 116). (Case E,F) Chest CT image sections from a 56 and 52-year-old men with confirmed COVID-19 on admission. Chest CT images showed bilateral subpleural and focal ground-glass density areas in the lower lobes more specifically. Their RT-PCR tests converted from negative to positive 3rd and 4th days after the first admission, respectively. 
TA B L E 4. Treatments, complication and outcomes of critically ill patients.

All patients $(\mathrm{N}=105) \quad$ Confirmed COVID-19 $(\mathrm{N}=27) \quad$ Suspected COVID-19 $(\mathrm{N}=78) \quad P$-value

Treatments

Drugs

Hydroxychloroquine

$94(89.5 \%)$

$27(100 \%)$

$67(85.9 \%)$

0.062

Oseltamivir

$30(28.6 \%)$

$5(18.5 \%)$

$25(32.1 \%)$

0.180

Favipiravir

$71(67.6 \%)$

$26(96.3 \%)$

$45(57.7 \%)$

$0.001 *$

Tocilizumab

7 (6.7\%)

7 (25.9\%)

$0(0 \%)$

$0.001 *$

Azithromycin

$71(67.6 \%)$

$21(77.8 \%)$

$50(64.1 \%)$

0.191

Vitamin C

37 (35.2\%)

$20(74.1 \%)$

$17(21.8 \%)$

$0.001 *$

Plasma therapy

$10(9.5 \%)$

$8(29.6 \%)$

$2(2.6 \%)$

$0.001 *$

Methylprednisolone

$17(16.2 \%)$

$11(40.7 \%)$

$6(7.7 \%)$

$0.001 *$

Antibiotic

$34(32.4 \%)$

$3(11.1 \%)$

$31(39.7 \%)$

$0.006^{*}$

Oxygen support

$\begin{array}{lcccc}\text { IMV } & 54(51.4 \%) & 15(55.6 \%) & 39(50 \%) & 0.619 \\ \text { NIMV } & 64(61.5 \%) & 18(66.7 \%) & 46(59.7 \%) & 0.524 \\ \text { HFNC } & 15(14.3 \%) & 5(18.5 \%) & 10(12.8 \%) & 0.527 \\ \text { Nasal cannula } & 6(5.7 \%) & 3(11.1 \%) & 3(3.8 \%) & 0.175 \\ \text { ECMO } & 0(0 \%) & 0(0 \%) & 0(0 \%) & 0.447 \\ \text { RRT } & 10(9.5 \%) & 1(3.7 \%) & 9(11.5 \%) & \end{array}$

Complications

$\begin{array}{lcccc}\text { ARDS } & 20(19 \%) & 11(40.7 \%) & 9(11.5 \%) & 0.001 * \\ \text { AKI } & 7(6.7 \%) & 2(7.4 \%) & 5(6.4 \%) & >0.99\end{array}$

Clinical outcomes

\begin{tabular}{lcccc} 
Death & $64(61 \%)$ & $21(77.8 \%)$ & $43(55.1 \%)$ & $0.038 *$ \\
Staying in hospital & $20(19 \%)$ & $3(11.1 \%)$ & $17(21.8 \%)$ & 0.223 \\
Discharge & $25(23.8 \%)$ & $6(22.2 \%)$ & $19(24.4 \%)$ & 0.822 \\
\hline
\end{tabular}

Abbreviation: AKI, acute kidney injury; ARDS, acute respiratory distress syndrome; ECMO, extracorporeal membrane oxygenation; HFNC, high-flow nasal cannula; RRT, renal replacement therapy; IMV, invasive mechanical ventilation; NIMV, non-invasive mechanical ventilation. *P values $<0.05$.

TA B L E 5. Comparison of determining parameters with c-CT findings for suspected COVID-19 patients.

\begin{tabular}{|c|c|c|c|c|}
\hline & All Patients $(\mathrm{N}=78)$ & $\begin{array}{l}\text { Patients with typical c- } \\
\text { CT findings }(\mathrm{N}=36)\end{array}$ & $\begin{array}{l}\text { Patients with atypical } \\
\text { c-CT findings }(\mathrm{N}=42)\end{array}$ & $P$-value \\
\hline $\mathrm{PaO}_{2} / \mathrm{FiO}_{2}$ ratio, $\mathrm{mmHg}$, mean $( \pm \mathrm{SD})$ & $191.5( \pm 82.2)$ & $170.9( \pm 74.4)$ & $209.1( \pm 854)$ & $0.038^{*}$ \\
\hline APACHE II, mean $( \pm$ SD $)$ & $24.62( \pm 7.24)$ & $26.5( \pm 7.87)$ & $23( \pm 6.31)$ & $0.034^{*}$ \\
\hline The number of Comorbidity, mean $( \pm \mathrm{SD})$ & $1.87( \pm 1.22)$ & $2.22( \pm 1.33)$ & $1.57( \pm 1.03)$ & $0.020 *$ \\
\hline Lymphocyte $\left(10^{3} / \mu \mathrm{L}\right)$, mean $( \pm \mathrm{SD})$ & $1.44( \pm 1.47)$ & $1.37( \pm 1.54)$ & $1.49( \pm 1.42)$ & 0.746 \\
\hline $\mathrm{D}$-dimer $(\mu \mathrm{g} / \mathrm{L})$, mean $( \pm \mathrm{SD})$ & $390( \pm 546)$ & $446( \pm 705)$ & $363( \pm 411)$ & 0.520 \\
\hline Ferritin $(\mathrm{ng} / \mathrm{mL})$, mean $( \pm \mathrm{SD})$ & $475.42( \pm 835.20)$ & $658.84( \pm 1112.59)$ & $318.20( \pm 445.51)$ & 0.720 \\
\hline ARDS & $9(11.5 \%)$ & $6(16.7 \%)$ & $3(7.1 \%)$ & 0.288 \\
\hline Death & $43(55.1 \%)$ & $24(66.7 \%)$ & $19(45.2 \%)$ & 0.058 \\
\hline
\end{tabular}

Abbreviation: ARDS, acute respiratory distress syndrome; APACHE II, Acute Physiology and Chronic Health

Evaluation II; $\mathrm{PaO}_{2} / \mathrm{FiO}_{2}$, ratio of partial pressure of arterial oxygen to fraction of inspired oxygen.

diseases such as COVID-19. The admission to ICUs depends on the severity of the disease in addition to the capacity of the health-care system. Treating infected critically ill patients in the same room as non-infected patients may increase the risk of transmission of the disease while treating non-infected patients in isolated rooms could result in unnecessary hospitalization 
and financial burden for ICUs with limited conditions. To our knowledge, this article is the first study to evaluate patients with suspected COVID-19 infection in terms of ICU triage.

Initial reports have shown that COVID-19 is associated with severe disease that requires intensive care in approximately $5 \%$ of infections [19]. In Huang's study, $32 \%$ of patients were admitted to the ICU, the median age was 49 (41-61), and the most common symptom was dyspnea. The most common complication was ARDS (85\%). Thirty-eight percent of patients died [10]. In another study, the mean age of 52 critically ill patients was 59.7 years, 35 (67\%) of whom were male. Twenty-one (40\%) had a chronic illness [20]. Murty et al. stated that there was a wide mortality range, from $22 \%$ to $62 \%$ in ICU [21]. In our study, the median age of 105 critically ill patients was $70(58.5-80.0)$. The most common symptom was shortness of breath $(81 \%)$. The most common complications were ARDS (19\%) and AKI (6.7\%), with ARDS being significantly higher in confirmed COVID-19 patients than in suspected COVID-19 patients. Of our patients, 64 $(61 \%)$ died, and the mortality rate in the confirmed COVID19 group was higher than in the suspected group.

2019-nCoV may be more likely to infect older men due to weaker immune function in patients with chronic comorbidities. Moreover, a comprehensive assessment of comorbidities may help establish risk classification of patients with COVID19 on hospital admission because such individuals might be more vulnerable to severe coronavirus infection [8]. In a study, patients admitted to ICU were older than patients who did not require admission to ICU, and had a high number of comorbid conditions. The most common comorbid diseases were hypertension (58.3\%) and diabetes $(21.6 \%)$. Accordingly, the authors stated that age and comorbidity might be risk factors for poor outcomes. Patients who required ICU care were more likely to have underlying comorbidities $(72.2 \%$ compared to $37 \%$ ) [11]. Guan et al. stated that the most common comorbid diseases were hypertension (23.7\%) and diabetes (16.2\%) in severe cases [22]. Of our patients, $96(91.4 \%)$ had a preexisting comorbid disease. Diabetes (36.2\%) and hypertension (29.5\%) were the most common comorbid diseases. A patient with immune disease required only oxygen support with a nasal cannula, but he died in the 48th hour of hospitalization in ICU. Interestingly, our data suggest that the number of preexisting comorbid diseases may be important in the diagnosis of COVID-19 infection rather than the presence of them.

The ratio of arterial to inspired $\mathrm{PO}_{2}\left(\mathrm{PaO}_{2} / \mathrm{FiO}_{2}\right)$ is one of the most common parameters used in admission to ICUs [23]. Moreover, the APACHE II scoring system has been employed primarily to predict patient outcomes and, only through these data, indirectly as a triage tool to predict which patients will require intensive care [24]. Wang et al. noted that the median $\mathrm{PaO}_{2} / \mathrm{FiO}_{2}$ ratio of patients in the ICU was $136 \mathrm{mmHg}$ (IQR, 103-234) and the APACHE II score was 17 (IQR, 10-22) [11]. Chen et al. reported that the median $\mathrm{PaO}_{2} / \mathrm{FiO}_{2}$ ratio was significantly lower in deceased patients $(105.1 \mathrm{mmHg})$ than in recovered patients (350.0 mmHg) [25]. The mean $\mathrm{PaO}_{2} / \mathrm{FiO}_{2}$ ratio of our patients was $182.5 \mathrm{mmHg}( \pm 80.8)$, and it was significantly lower in confirmed COVID-19 patients. The mean APACHE II score of our patients was 25.55 ( \pm 7.92$)$, and it was significantly higher in confirmed COVID-19 cases.
Additionally, we noted that compared to suspected COVID-19 patients with atypical c-CT findings, $\mathrm{PaO}_{2} / \mathrm{FiO}_{2}$ ratios were lower $[170.9( \pm 74.4)]$ in suspected patients with typical c-CT findings. Also, compared to suspected patients with atypical cCT findings, mean APACHE II scores were higher in suspected patients with typical c-CT findings. These data may suggest that suspected patients with typical c-CT findings should be reevaluated for a diagnosis of COVID-19 infection.

In vitro cell experiments have shown that the release of cytokines and chemokines occurs in respiratory epithelial cells and macrophages in the early stage of SARS-CoV infection, and a cytokine storm is induced. Later changes in peripheral white blood cells and immune cells such as lymphocytes also occurred [26]. Yang et al. detected lymphopenia in more than $80 \%$ of critically ill patients in their studies [20]. Wang et al. stated that most patients had marked lymphopenia during hospitalization, and non-survivors developed more severe lymphopenia over time. Additionally, the median D-dimer value was 414 (191-1324) $\mu \mathrm{g} / \mathrm{L}$ in ICU patients, and they stated that this was significantly higher than in non-ICU patients [11]. In our research, lymphocyte values were significantly low [0.98 $( \pm 0.51)]$ in confirmed COVID-19 cases. The lymphocyte values were lower in suspected COVID-19 patients with typical c-CT findings than those with atypical c-CT findings, but this was not statistically significant. In our patients, the mean D-dimer values $[350( \pm 501) \mu \mathrm{g} / \mathrm{L}]$ were normal range and serum ferritin values $[528.49( \pm 748.23) \mathrm{ng} / \mathrm{mL}]$ were above the normal range.

There is no proven therapy for COVID-19, but several drugs used against SARS-CoV and MERS CoV have been used empirically, and new drug research is proceeding [27]. Currently, the optimal approach for this disease is to control the source of infection. The using personal protective equipment to reduce the risk of transmission, early diagnosis, isolation, and supportive therapy are essential practices for affected patients. Arabi et al. expressed that usage rate of IMV was 42\% [28]. Reports indicate that NIMV and HFNC are used in between one-third and twothirds of critically ill patients with COVID-19 in ICU in China [29]. In our study, patients received NIMV, IMV, HFNC and oxygen only via a simple nasal cannula $(61.5 \%$, $51.4 \%, 14.3 \%$, and $5.7 \%$, respectively). ECMO was not applied to any patient. To date, no specific treatment has been recommended for COVID-19 infection, except for rigorous supportive care [30]. However, various treatment regimens have been used, such as remdesivir [31], lopinavirritonavir (internal) chloroquine [32], hydroxychloroquine and azithromycin (33.34), tocilizumab [35], and favipiravir [36]. Most of our patients received hydroxychloroquine (89.5\%), oseltamivir (28.6\%), favipiravir $(67.6 \%)$, azithromycin (67.6\%), tocilizumab (6.7\%), immune plasma (9.5\%), vitamin C (35.2\%), methylprednisolone (16.2\%), and antibiotic therapy $(32.4 \%)$.

Currently, RT-PCR amplification of the viral RNA is considered the "gold standard" in the diagnosis of COVID-19. However, the first RT-PCR sample may not always be positive in patients with COVID-19 infection [37,38]. RT-PCR testing results may be affected by many factors. External factors include sampling procedures, place of specimens, sampling 
timing, and performance of detection kits. In addition to RTPCR, c-CT has been widely used in the diagnosis of coronavirus pneumonia since it was found to be highly sensitive for COVID-19. Multiple patchy ground-glass opacities in bilateral multiple lobular with periphery distribution are typical c-CT imaging features of COVID-19 pneumonia [6]. Of our critical patients, 63 (60\%) had typical c-CT findings for COVID-19, and all confirmed cases had typical c-CT findings. In a study on the correlation of c-CT and RT-PCR, the sensitivity of c-CT imaging for COVID-19 was 97\%. Following a comprehensive reevaluation of patients with negative RT-PCR results but positive c-CT scans, $48 \%$ were considered as highly likely cases, with $33 \%$ considered probable cases. As a result of these findings, the authors stated that in patients with negative RT-PCR tests, a combination of clinical symptoms, preexisting diseases, typical c-CT imaging features, and dynamic changes should be used to identify COVID-19 with higher sensitivity [37]. Some studies even stated that c-CT is more sensitive than RT-PCR. In Long's study, c-CT sensitivity was $97.2 \%$, whereas the sensitivity of initial RT-PCR was only $83.3 \%$. cCT examinations appear sensitive to virus detection, whereas RT-PCR may produce false-negative results [39]. In our study, c-CT findings for all confirmed COVID-19 cases (27, 100\%) were compatible with typical c-CT findings and none of the patients with atypical c-CT findings $(0,100 \%)$ had a positive PCR test. Accordingly, we considered that c-CT imaging might be an important measurement tool after RT-PCR testing for a new triage system.

In our ICUs, four cases missed by the RT-PCR testing had earlier been detected by the c-CT examination on the first admission. Of patients with a negative PCR test, $46.2 \%$ had typical c-CT findings for COVID-19. We used the c-CT examination as a second diagnostic tool to reevaluate the suspected cases based on both typical and atypical c-CT findings. In suspected cases, when compared with patients with atypical cCT findings, the patients with typical c-CT findings had lower $\mathrm{PaO}_{2} / \mathrm{FiO}_{2}$ ratios, higher APACHE II scores, and more preexisting comorbid diseases. This shows that in cases with negative RT-PCR testing, a patient's $\mathrm{PaO}_{2} / \mathrm{FiO}_{2}$ ratio, APACHE II score, and the number of preexisting comorbid diseases in addition to typical c-CT findings may be useful in the early diagnosis of COVID-19. If these parameters were taken into consideration, $33.3 \%$ of cases with typical c-CT findings could be reconsidered as highly probable COVID-19 infections. Moreover, if these parameters were considered, $16.7 \%$ of the cases with atypical c-CT findings could be excluded in terms of COVID-19 infection, and the unnecessary burden on ICUs could be reduced. Hence, during the admission of patients who have negative RT-PCR test and typical c-CT imaging for COVID-19, the low $\mathrm{PaO}_{2} / \mathrm{FiO}_{2}$ ratio, the higher APACHE II score and the number of preexisting comorbid diseases might be some of the substantial parameters, which are needed to be used in a new triage system.

This study had some limitations. Firstly, only 105 critically ill patients were included, making the sample size of this study small. Research involving more patients will be required for further verification. The secondary aim of the study was to reveal that a triage system is needed to determine the indications for admission to ICUs for contagious diseases. Therefore, some specific scoring parameters according to the type of the disease are required.

In conclusion, a pandemic may lead to a critical increase in the need for ICU beds. If an ICU is overwhelmed by COVID-19 or any other contagious disease despite intensive care strategies, a new intensive care triage system that prioritizes patients with the correct hospitalization criteria will be required. For a new triage system, specific characteristics, selected general physiological findings, and typical laboratory parameters may be standardized in addition to RT-PCR testing and c-CT examination. Further studies will be required to more precisely determine these parameters in the future.

\section{AUTHOR CONTRIBUTIONS}

MAY and YT designed the study. FY, HS and EK collected the data. FY, EK, NS and GY analyzed the data. MAY, YT, HS, NS and GY analyzed the results and drafted the manuscript.

\section{ETHICS APPROVAL AND CONSENT TO PARTICIPATE}

This retrospective study was approved by the local ethics committee of Selcuk University, Konya (Acceptance number: 2020/245).

\section{ACKNOWLEDGMENT}

The authors thank ICU staffs who have made unstintingly their effort for ICU patients with coronavirus in Konya Training and Research Hospital for supporting the collection of data.

\section{FUNDING}

This research received no external funding.

\section{CONFLICT OF INTEREST}

The authors declare no conflict of interest.

\section{REFERENCES}

[1] World Health Organization. Novel coronavirus (2019-nCoV): situation report-119. 2020. Available at: https://www.who.int/ docs/default-source/coronaviruse/situation-reports/ 20200518-covid-19-sitrep-119.pdf?sfvrsn=4bd9de25_4 (Accessed: 18 May 2020).

[2] Chan JF, Yuan S, Kok K, To KK, Chu H, Yang J, et al. A familial cluster of pneumonia associated with the 2019 novel coronavirus indicating personto-person transmission: a study of a family cluster. Lancet. 2020; 395 : 514-523.

[3] Rothe C, Schunk M, Sothmann P, Bretzel G, Froeschl G, Wallrauch C, et al. Transmission of 2019-nCoV infection from an asymptomatic contact in Germany. New England Journal of Medicine. 2020; 382: 970-971.

[4] Wang Z, Yang B, Li Q, Wen L, Zhang R. Clinical features of 69 cases with coronavirus disease 2019 in Wuhan, China. Clinical Infectious Diseases. 2020; 71: 769-777.

[5] Chen L, Liu H, Liu W, Liu J, Liu K, Shang J, et al. Analysis of clinical features of 29 patients with 2019 novel coronavirus pneumonia. Chinese Journal of Tuberculosis and Respiratory Diseases. 2020; 43: 203-208. (In Chinese) 
[6] Xu X, Yu C, Qu J, Zhang L, Jiang S, Huang D, et al. Imaging and clinical features of patients with 2019 novel coronavirus SARS-CoV-2. European Journal of Nuclear Medicine and Molecular Imaging. 2020; 47: 12751280 .

[7] Chen N, Zhou M, Dong X, Qu J, Gong F, Han Y, et al. Epidemiological and clinical characteristics of 99 cases of 2019 novel coronavirus pneumonia in Wuhan, China: a descriptive study. Lancet. 2020; 395: 507-513.

[8] Bansal Garg I, Srivastava S, Rai C, Kumar V, Hembrom A, Ghosh N, et al. Coronavirus (COVID-19): prognostic risk associated with comorbidities and age. International Journal of Recent Scientific Research. 2020; 37983-37986.

[9] Fehr AR, Channappanavar R, Perlman S. Middle east respiratory syndrome: emergence of a pathogenic human coronavirus. Annual Review of Medicine. 2017; 68: 387-399.

[10] Huang C, Wang Y, Li X, Ren L, Zhao J, Hu Y, et al. Clinical features of patients infected with 2019 novel coronavirus in Wuhan, China. Lancet. 2020; 395: 497-506.

[11] Wang D, Hu B, Hu C, Zhu F, Liu X, Zhang J, et al. Clinical characteristics of 138 hospitalized patients with 2019 novel coronavirusinfected pneumonia in Wuhan, China. The Journal of the American Medical Association. 2020; 323: 1061-1069.

[12] World Health Organization. Laboratory testing for 2019 novel coronavirus (2019-nCoV) in suspected human cases. 2020. Available at: https://www. who. int/publications-detail/laboratorytesting-for-2019-novel-coronavirus-in-suspectedhuman-cases-20200117 (Accessed: 17 May 2020).

[13] Chung M, Bernheim A, Mei X, Zhang N, Huang M, Zeng X, et al. CT imaging features of 2019 novel coronavirus (2019-nCoV). Radiology. 2020; 295: 202-207.

[14] Ranieri VM, Rubenfeld GD, Thompson BT, Ferguson ND, Caldwell E, Fan E, et al. Acute respiratory distress syndrome: the Berlin definition. The Journal of the American Medical Association. 2012; 307: 25262533.

[15] Kidney Disease: Improving Global Outcomes (KDIGO) Acute Kidney Injury Work Group. KDIGO Clinical practice guideline for acute kidney injury. KDIGO. 2012; 2: 1-138.

[16] Hansell DM, Bankier AA, MacMahon H, McLoud TC, Müller NL, Remy J. Fleischner Society: glossary of terms for thoracic imaging. Radiology. 2008; 246: 697-722.

[17] Shi H, Han X, Jiang N, Cao Y, Alwalid O, Gu J, et al. Radiological findings from 81 patients with COVID-19 pneumonia in Wuhan, China: a descriptive study. Lancet Infectious Diseases. 2020; 20: 425-434.

[18] Pan F, Ye T, Sun P, Gui S, Liang B, Li L, et al. Time course of lung changes at chest CT during recovery from coronavirus disease 2019 (COVID-19). Radiology. 2020; 295: 715-721.

[19] Wu Z, McGoogan JM. Characteristics of and important lessons from the Coronavirus Disease 2019 (COVID-19) outbreak in China: summary of a report of 72,314 cases from the Chinese center for disease control and prevention. The Journal of the American Medical Association. 2020; 323 : 1239-1242.

[20] Yang X, Yu Y, Xu J, Shu H, Xia J, Liu H, et al. Clinical course and outcomes of critically ill patients with SARS-CoV-2 pneumonia in Wuhan, China: a single-centered, retrospective, observational study. Lancet Respiratory Medicine. 2020; 8: 475-481.

[21] Murthy S, Gomersall CD, Fowler RA. Care for critically ill patients with COVID-19. The Journal of the American Medical Association. 2020; 323: 1499.

[22] Guan W-J, Liang W-H, Zhao Y, Liang H-R, Chen Z-S, Li Y-M, et al. Comorbidity and its impact on 1590 patients with COVID-19 in China: a nationwide analysis. The European Respiratory Journal. 2020; 55: 2000547.

[23] Wagner PD. Ventilation/perfusion relationships. In Hamid, Shannon,
Martin (eds.) Physiological basis of respiratory disease (pp. 164-184). USA: People's Medical Publishing House. 2005.

[24] Franklin C. Triage considerations in medical intensive care. Archives of Internal Medicine. 1990; 150: 1455-1459.

[25] Chen T, Wu D, Chen H, Yan W, Yang D, Chen G, et al. Clinical characteristics of 113 deceased patients with coronavirus disease 2019: retrospective study. British Medical Journal. 2020; 368: m1091.

[26] Ye Q, Wang B, Mao J. The pathogenesis and treatment of the 'Cytokine Storm' in COVID-19. Journal of Infection. 2020; 80: 607-613.

[27] World Health Organization. Clinical management of severe acute respiratory infection (SARI) when COVID-19 disease is suspected: interim guidance. 2020. Available at: https://www.who.int/ publications-detail/clinicalmanagement-of-severeacute-respiratory-infection-when-novelcoronavirus(ncov)-infection-is-suspected (Accessed: 15 March 2020).

[28] Arabi YM, Murthy S, Webb S. COVID-19: a novel coronavirus and a novel challenge for critical care. Intensive Care Medicine. 2020; 46: 833836.

[29] Phua J, Weng L, Ling L, Egi M, Lim C, Divatia JV, et al. Intensive care management of coronavirus disease 2019 (COVID-19): challenges and recommendations. Lancet Respiratory Medicine. 2020; 8: 506-517.

[30] de Wit E, van Doremalen N, Falzarano D, Munster VJ. SARS and MERS: recent insights into emerging coronaviruses. Nature Reviews Microbiology. 2016; 14: 523-534.

[31] Sheahan TP, Sims AC, Leist SR, Schäfer A, Won J, Brown AJ, et al. Comparative therapeutic efficacy of remdesivir and combination lopinavir, ritonavir, and interferon beta against MERS-CoV. Nature Communications. 2020; 11: 222.

[32] Wang M, Cao R, Zhang L, Yang X, Liu J, Xu M, et al. Remdesivir and chloroquine effectively inhibit the recently emerged novel coronavirus (2019-nCoV) in vitro. Cell Research. 2020; 30: 269-271.

[33] Yao X, Ye F, Zhang M, Cui C, Huang B, Niu P, et al. In vitro antiviral activity and projection of optimized dosing design of hydroxychloroquine for the treatment of severe acute respiratory syndrome coronavirus 2 (SARS-CoV-2). Clinical Infectious Diseases. 2020; 71: 732-739.

[34] Gautret P, Lagier JC, Parola P, Hoang VT, Meddeb L, Mailhe M, et al. Hydroxychloroquine and azithromycin as a treatment of COVID19: results of an open-label non-randomized clinical trial. International Journal of Antimicrobial Agents. 2020; 56: 105949.

[35] Chen C, Zhang XR. Advances in the research of cytokine storm mechanism induced by corona virus disease 2019 and the corresponding immunotherapies. Chinese Journal of Burns. 2020; 36: E005.

[36] Cai Q, Yang M, Liu D, Chen J, Shu D, Xia J, et al. Experimental treatment with favipiravir for COVID-19: an open-label control study. Engineering. 2020; 6: 1192-1198.

[37] Ai T, Yang Z, Hou H, Zhan C, Chen C, Lv W, et al. Correlation of chest CT and RT-PCR testing for coronavirus disease 2019 (COVID-19) in China: a report of 1014 cases. Radiology. 2020; 296: E32-E40.

[38] Fang Y, Zhang H, Xie J, Lin M, Ying L, Pang P, et al. Sensitivity of chest CT for COVID-19: comparison to RT-PCR. Radiology. 2020; 296: E115E117.

[39] Long C, Xu H, Shen Q, Zhang X, Fan B, Wang C, et al. Diagnosis of the Coronavirus disease (COVID-19): rRT-PCR or CT? European Journal of Radiology. 2020; 126: 108961.

How to cite this article: Mehmet Akif YAZAR, Yasin TIRE, Fatih YUCEL, Hasan SENAY, Ercan KURTIPEK, Nevin SEKMENLI. Clinical characteristics of critically Ill patients with 2019 novel coronavirus (COVID-19): do we need a new triage system? Signa Vitae. 2021;17(3):121-129. doi:10.22514/sv.2021.066. 